\title{
Electron Beam as a Probe and Stimulus: Challenges and Opportunities
}

\author{
Sadegh Yazdi
}

Renewable and Sustainable Energy Institute, University of Colorado Boulder, Boulder, Colorado, United States

The transmission electron microscope (TEM) is one of the most potent characterization tools in materials science. The electron beam in TEM is usually used as an imaging or spectroscopy probe for chemical and physical characterization of materials and nanostructures. In many TEM experiments, however, besides the inherent characteristics of the material, the electron beam reveals the physical or chemical reactions stimulated by the electron beam itself. Usually, such electron beam-induced reactions are considered undesired beam effects/damage that needs to be suppressed. High energy electrons can displace atoms, electrically charge the material, raise the specimen temperature, and ionize and dissociate molecules — reactions that need to be mitigated. Despite being undesirable in characterizing TEM specimens, such beam-induced phenomena can lead to novel applications if understood and controlled. For example, the technique of electron beam lithography owes its existence to the ability of an electron beam to transform/de-cross-link polymers, which is considered beam damage in imaging polymers in the TEM. The electron beam is capable of probing the same physical or chemical reactions that are stimulated by itself, with high spatial and energy resolution. As examples, we will present a few such experiments and discuss the challenges and opportunities in employing this capability in the TEM.

The radiolysis of liquids under the electron irradiation is generally viewed as a hurdle in developing in-situ liquid phase TEM. We, nevertheless, took advantage of this electron beam-induced reaction to reshape the interior structure of $\mathrm{AgAu}$ nanorods (see ref [1] for details). In synthesizing AgAu nanorods by the galvanic replacement method, a pocket of the aqueous solution can be encapsulated inside the hollow structure of the nanorods. Therefore, these nanorods become a perfect closed liquid cell for in-situ TEM studies. Irradiating the trapped water with the electron beam generates radiolytic species including solvated electrons and hydroxyl radicals, strong reducing and oxidizing agents, respectively. In the scanning TEM (STEM) mode, we illuminate a small part of the liquid medium with a high electron dose to generate enough solvated electrons to reduce Ag ions to Ag near the electron beam. This results in the migration of Ag to the region under the electron beam and therefore reshapes the nanorod interior structure. Simultaneously, the generated hydroxyl radicals oxidize Ag to Ag ions away from the beam. We switch between the probing and stimulating modes by controlling the electron dose in the STEM mode. Reshaping the internal structure of AgAu nanorods also changes their optical/plasmonic properties. We used monochromated electron energy loss spectroscopy (EELS) to correlate nanorods' internal structures with their localized surface plasmon resonance (LSPR) properties (Fig. 1). Mapping LSPR with EELS can also be viewed as another example of using the electron beam to stimulate and probe a physical phenomenon, in this case, exciting LSPR.

Another experimental example where the electron beam is employed to modify the property of materials is shown in Fig. 2. By carrying out in-situ heating experiments in TEM on helical nanofilaments (HNF), a chiral liquid crystal, we found that irradiating the HNF with the electron beam increases its phase transition temperatures. The annular dark field (ADF) STEM image of HNFs in Fig. 2a, acquired at $300 \mathrm{kV}$ and room temperature, clearly reveals the twisted smectic layers. Above $\sim 170{ }^{\circ} \mathrm{C}$, the $\mathrm{HNF}(\mathrm{B} 4)$ and other Bx phases (B1-B8) change to the isotropic liquid phase [2]. However, after irradiating the HNF sample with the electron beam, we could image the HNF at significantly higher temperatures. Fig. 2c shows an ADF STEM image of $\mathrm{HNFs}$ at $700{ }^{\circ} \mathrm{C}$ as an example. The electron beam-induced polymerization is likely to be responsible for the stability of HNFs at high temperatures, and much improved thermal stability of HNFs might open avenues for new applications. 

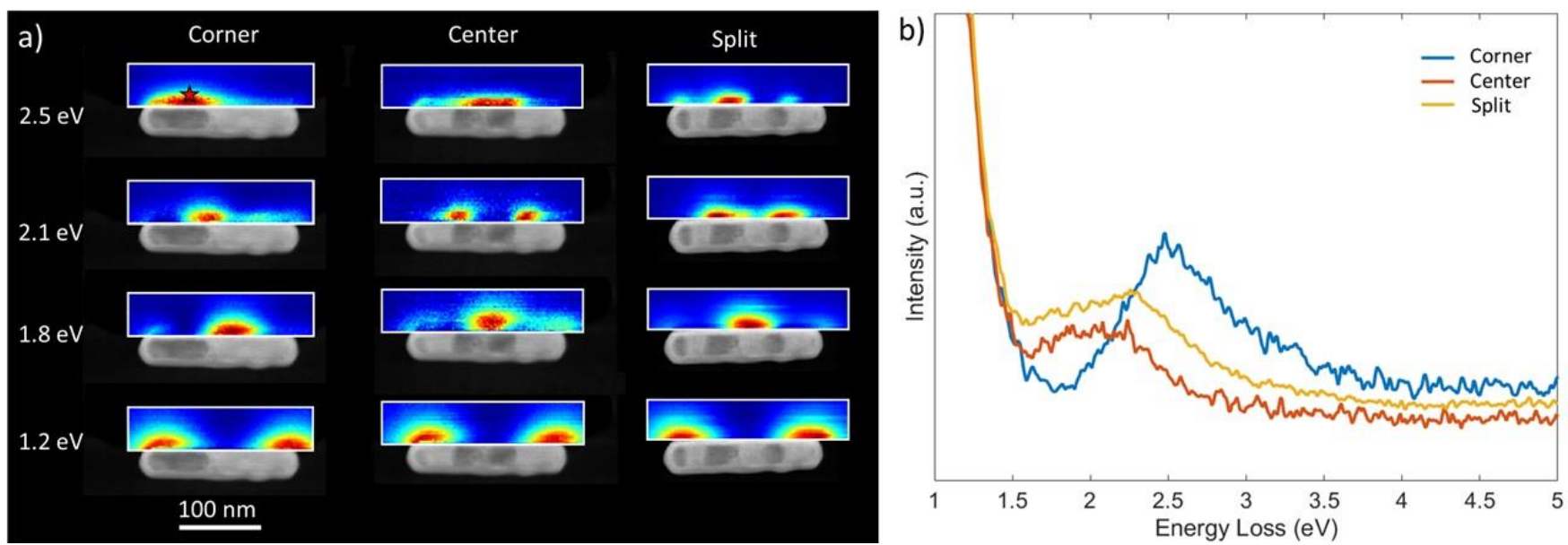

Figure 1. Fig. 1: Reshaping the interior structure of $\mathrm{AgAu}$ nanorods by radiolysis of encapsulated aqueous solutions in their hollow structure using the electron irradiation in TEM and mapping their corresponding plasmonic responses using monochromated STEM-EELS. a) The same AgAu nanorod with three different interior structures (corner, center, and split) shaped by the electron beam and their corresponding LSPR maps at 2.5, 2.1, 1.8, and 1.2 eV. b) EELS spectra from a single position, marked by the red star in a), for the corner, center, and split structures showing the difference in their optical response. The figure is adapted from reference [1]. 


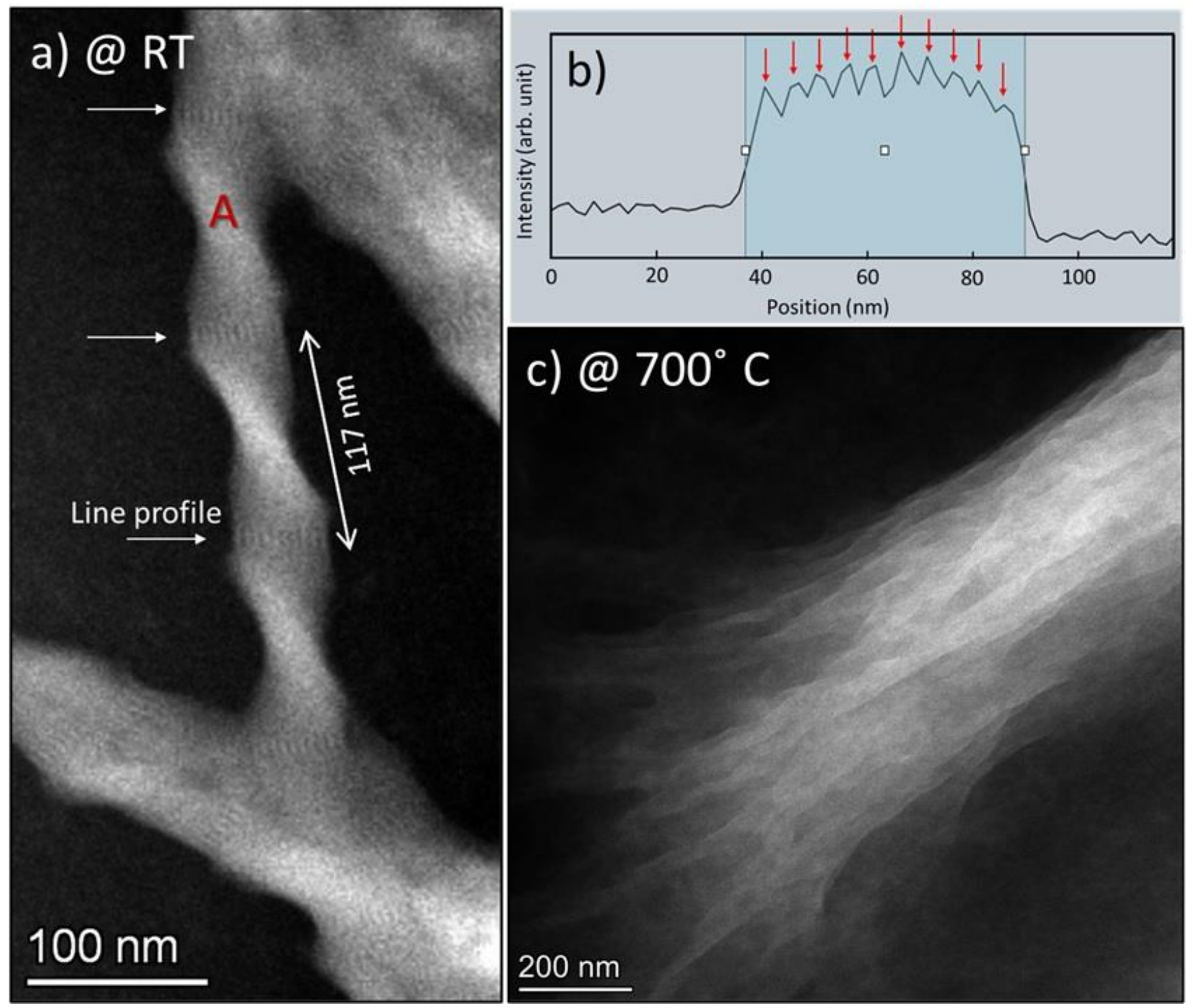

Figure 2. Fig. 2: a) ADF STEM image of a helical nanofilament (HNF) acquired at room temperature at 300 $\mathrm{kV}$. The white arrows point where the twisted smectic layers are aligned perpendicular to the electron beam, and therefore, they are visible. b) Line profile across filament A showing ten smectic layers for this filament. The measured distance between the layers is $5.3 \mathrm{~nm}$, and the filament thickness is $53 \mathrm{~nm}$. The red arrows indicate different layers. c) ADF STEM image of a bundle of helical nanofilaments acquired at $700^{\circ} \mathrm{C}$, significantly above HNF's transition temperature to the liquid phase.

\section{References}

[1] S. Yazdi, J. R Daniel, N Large, G. C Schatz, D. Boudreau, and E. Ringe, Nano Letters., 2016, 16, 39396945.

[2] L. E. Hough, H. T. Jung, D. Krüerke, M. S. Heberling, M. Nakata, C. D. Jones, D. Chen, D. R. Link, J. Zasadzinski, G. Heppke, J. P. Rabe, W. Stocker, E. Körblova, D. M. Walba, M. A. Glaser, N. A. Clark, Science, 2009, 325, 456-460

[3] The support from the Facility for Electron Microscopy of Materials at the University of Colorado at Boulder (CU FEMM) is acknowledged. 